\title{
Die ersten skandinavischen Bibelübersetzungen und ihre soziokulturellen Auswirkungen
}

\author{
Attila KELEMEN \\ Sapientia Hungarian University of Transylvania \\ Faculty of Technical and Human Sciences \\ Department of Applied Linguistics, Târgu Mureş \\ akelemen20@yahoo.no
}

Zusammenfassung. Die vorliegende Abhandlung befasst sich mit der Entstehung der ersten vollständigen Bibelübersetzungen in den skandinavischen Sprachen, sowie mit deren soziokulturellen Auswirkungen. Indem wir die vergleichende Methode verwenden und uns der Forschungsergebnisse linguistischer Fächer, wie Sprachgeschichte, Soziolinguistik und Sprachkontaktforschung, aber auch anderer Wissenschaftszweige, wie Geschichte und Kulturgeschichte bedienen, versuchen wir zu beweisen, dass, trotz der Ähnlichkeiten der skandinavischen Sprachen, die verschiedenen historisch-politischen Umständen zu unterschiedlichen Entwicklungen hinsichtlich der Nationalsprachen und folglich der nationalen Kulturen geführt haben. In diesem Zusammenhang erwies sich ausschlaggebend auch die Übersetzung der Bibel in die Nationalsprachen zu unterschiedlichem Zeitpunkt.

Schlüsselwörter: Reformation, Bibelübersetzung, Nationalsprache, Schrifttradition, Schriftsprache

\begin{abstract}
The first Scandinavian Bible translations and their socio-cultural impact. The present paper deals with the first complete Bible translations into the Scandinavian languages and with the socio-cultural impact of these. Using the comparative method and making use of the research results of linguistic disciplines like language history and sociolinguistics, but also of other disciplines like history and cultural history, we try the prove that, in spite of the similarities of the Scandinavian languages, the different historical-political circumstances lead to differing evolution of the national languages, and implicitly of the national cultures. In this context, the translation of the Bible into the national languages at different point in time turned out to be decisive.
\end{abstract}

Keywords: Reformation, Bible translation, national language, writing tradition, written language 
I. Die vorliegende Abhandlung befasst sich mit der Entstehung der ersten vollständigen skandinavischen Bibelübersetzungen, mit den geschichtlichen Umständen, in denen diese zustande gekommen sind, sowie mit den soziokulturellen Auswirkungen dieser Übersetzungen.

Es wird untersucht, inwiefern die verschiedenen historisch-politischen Umstände zu unterschiedlichen Entwicklungen führten im Falle der skandinavischen Sprachen, die einander so ähnlich waren und sind, und inwieweit der Zeitpunkt der ersten Bibelübersetzungen für die Herausbildung der Nationalsprachen und der nationalen Kulturen entscheidend war.

Die Reformation war ein Wendepunkt in der Geschichte und führte zu einer Umwälzung in vielen Ländern. Als Martin Luther 1517 in Wittenberg seine Thesen veröffentlichte und damit seine Reformation in Deutschland ankündigte, waren im Nord-Europa zwei Königreiche im erbitterten Kampf miteinander um die Herrschaft in der Region: Dänemark, die stärker und mächtiger war, und sein Erzrivale, Schweden. In ihren Bestrebungen, ihre Herrschaft zu verstärken, „,bedienten sich die beiden derselben Maßnahmen, nämlich die Auflösung der katholischen Kirche, die den Königen ständig Rivalität leistete, und die Einführung der lutherischen Staatskirche unter königlichen Kontrolle” (Vikør 1993: 24).

II. Im späten Mittelalter drohte Dänemark nicht nur politisch. Auch die dänische Sprache „drohte die anderen mit ihrer Herrschaft zu überwältigen; Schwedisch entkam mit nur wenigen, während die norwegische Schriftsprache völlig beseitigt wurde; Färöisch und Isländisch sind trotz des starken Widerstands vom dänischen Einfluss deutlich markiert worden.” (Haugen 1976: 64)

Zuvor war die Mehrzahl der Schriften in Dänemark und Schweden auf lateinisch, und die ersten Schriftdenkmäler in den Nationalsprachen wurden vom Latein geprägt. Die frühesten Werke im 13. Jahrhundert zeugen davon, dass es, als die schwedische Schrifttradition etabliert wurde, nur kleine Unterschiede von der damaligen dänischen Schrift gab.

Als der dänische König Christian II die Schweden besiegte und sich 1520 in Stockholm zum König krönen ließ, veranstaltete er auch ein Blutbad unter den schwedischen Adeligen, wovon nur Gustav Vasa entfliehen konnte. Ein Jahr später stiftete Vasa einen Aufruhr, dann mit Hilfe der Hansestadt Lübeck gelang es ihm, die Dänen aus dem Lande zu verjagen. 1523 wurde er zum König. Die ersten Maßnahmen waren die Abschaffung der katholischen Kirche und die Beschlagnahme deren Eigentümer. Dann machte Gustav Vasa den Protestantismus zur Staatsreligion und sich zum Oberhaupt der Schwedischen Kirche.

Die Gründung des unabhängigen Schwedens kündigte den Anfang eines neuen Zeitalters für das Land sowohl politisch als auch kulturell. Schweden entwikkelte sich bald zu einem modernen Staat, ein Jahrhundert später galt es schon als europäische Großmacht. Die Einführung der Reformation förderte die Herausbil- 
dung der Nationalsprache. Mit der Übersetzung der Bibel (1541) ins Schwedische war die Grundlage für die moderne einheitliche schwedische Schriftsprache gelegt, die von nun an ihre eigene Entwicklung haben sollte.

Zuerst erschien die Übersetzung des Neuen Testaments (1526). Die Arbeit wurde unter der Leitung von Olaus Petri (Olof Persson) und Laurentius Andreae (Lars Andersson) ausgeführt. Diese Übersetzung folgte eine zwei Jahre zuvor erschienene dänische. Die Übersetzer haben sich darum bemüht, die vielen Danizismen zu beseitigen (rige wurde zu rike, tale zu tala). Morpho-syntaktisch war der Stil einfacher, moderner als der der 15 Jahre später erschienenen Bibel. Die Übersetzung des Neuen Testaments bedurfte einer gründlichen Revision, ehe sie in die 1541 erschienene Übersetzung der ganzen Bibel einverleibt werden konnte. Diese Übersetzung wurde als Gustav Vasas Bibel bekannt und wurde zur offiziellen Version. Die Übersetzungsarbeit wurde unter der persönlichen Überwachung des ersten lutherischen Erzbischofs von Uppsala, Laurentius Petri (Lars Persson), Bruder von Olaus Petri.

Laurentius Petri war ein gewissenhafter und konsequenter Sprachreformator, ein eifriger Anhänger der Sprachreinigung. In der Kyrkoordning „Kirchenregel“ aus dem Jahre 1571 forderte er die Priester und die Lehrer auf, Fremdwörter zu vermeiden und ihren Schülern „gute alte schwedische Wörter“ beizubringen.

Die meisten Philologen, wie z. B. Sjögren (1949), sind der Ansicht, dass die Übersetzer der Vasa-Bibel Luthers deutsche Übersetzung aus dem Jahre 1522 als Hauptmuster hatten, aber es gibt auch Meinungen, dass die mittelalterlichen Vadstena-Übersetzungen und die anderen früheren Versuche als Vorbild dienten; daher eine „archaische Version“ der Bibel. Die Sprache der Übersetzung ist archaisch, basiert auf die ältere Tradition, aber das war eben die Absicht der Reformatoren, sie wollten nämlich archaisieren. Laut Sjögren (1949: 146) sei die Sprache der Bibel „konservativ und traditionell“, und nicht archaisch. Hier muss man erwähnen, dass Teile der Bibel schon vor der Reformation übersetzt wurden, zwölf Bücher des Alten Testaments aus der Vulgata (Ende des 15. Jahrhunderts), sowie eine Auslese aus dem Pentateuch (rund 1350).

Sowohl im Vorwort des Neuen Testaments (1526) als auch in dem der Bibel (1541) wurde unterstrichen, der Text müsse „den armen Christen hier in diesem Königreich“ zugänglich gemacht werden, da diese an den Originaltexten nicht teilhaben können. Die Übersetzer stießen oft auf Schwierigkeiten, da es nicht immer möglich war, „,solche Wörter in der schwedischen Sprache zu finden, die den lateinischen und griechischen Wörtern gerecht werden konnten“ (Haugen 1976: 327).

Die Sprache der Bibelübersetzung fußte auf der gesprochenen Sprache MittelSchwedens, und insbesondere der der Provinz Uppland, der Region am Mälarsee, wo sich die Hauptstadt Stockholm und Uppsala, das Zentrum der Bildung (hier wurde die erste Universität Nord-Europas gegründet) befanden. Von hier 
stammten die Übersetzer. Auf die Mundarten dieser Region wird sich dann auch die spätere schwedische Standardsprache gründen.

Die Übersetzer hatten auch eine gewisse Normierung der Rechtschreibung vor, die dann langfristig als Vorbild für die Schriftsprache dienen sollte. So wurde in Gustav Vasas Bibel eine ziemlich konsequente Norm und Rechtschreibung entwickelt. Regiert von antidänischen Gefühlen, haben die Übersetzer in vielen Punkten gerade diejenigen Formen gewählt, die von den dänischen abwichen, dann auch die Unterschiede so viel wie möglich hervorgehoben. Sie nahmen sich der Adoption der Buchstaben $\ddot{a}, \ddot{o}$, ̊̊an, für die Laute, die im Dänischen die Schriftbilder æ, $\varnothing, a a$ hatten, dann bestärkten den Gebrauch des Ende- $a$ (ein Kennzeichen, das Schwedisch vom Dänisch und Norwegisch differenziert), obendrein wurden die alten Vollvokale wiedereingeführt, auch wenn sie schon geschwächt wurden. Viele alte Kasusformen (Dativ- und Akkusativendungen, redundanter Genitiv) wurden in der Übersetzungen bewahrt, obwohl sie in der gesprochenen Sprache schon als veraltet galten und sehr bald wegfielen, denn der Übergang von synthetischer zur analytischen Sprache hatte sich im Spätmittelalter im Großen und Ganzen vollzogen. Der Satzbau wurde von der lateinischen Syntax stark beeinflusst, und viele Fremdwörter wurden gebraucht.

Gustav Vasas Bibel freute sich weiter Verbreitung, und dazu trug wesentlich auch die Tatsache bei, dass der Buchdruck in Schweden schon im Jahre 1483 eingeführt worden war. Vasas Bibel diente dann lange als Vorbild für die Schriftnorm. Die religiöse Literatur wurde stilistisch von den Bibelübersetzungen beeinflusst.

Diese Bibelübersetzungen, insbesondere Gustav Vasas Bibel, lieferten die Grundlage für die moderne schwedische Schriftsprache und trugen nachdrücklich zur Entwicklung der nationalen Literatur und, implizit, der nationalen Kultur bei.

III. Die lutherische Reformation kam sehr zustatten auch den dänischen Königen, die ihre Macht der Kirche gegenüber verstärken wollten. Eifrige Prediger, wie Hans Tavsen, wurden ermutigt, ihre reformatorische Tätigkeit zu intensivieren. Im Laufe eines Jahrzehnts sind dann Priester, Mönche und andere Vertreter der katholischen Kirche aus dem Lande verjagt worden.

Tavsen, der als „der dänische Luther“ betrachtet wurde, übersetzte nun den Pentateuch, die fünf Bücher Mose ins Dänische. Er war auch an der Erarbeitung der neuen Kirchenordnung und der Reformation an der Kopenhagener Universität beteiligt. Als Belohnung wurde er zum Bischof von Ribe ernannt.

Der lutherische Protestantismus wurde offiziell zur Staatsreligion mit der Gründung der Dänischen Staatskirche im Jahre 1537, und nicht nur in Dänemark, sondern auch in den unter dänischer Herrschaft befindenden Gebieten. Dänisch verstärkte und befestigte seine Position, übernahm völlig die Rolle des Lateins in der Kirche, indem es zur Sprache der Gottesdienste und der Bibel wurde. 
Als die Niederschreibung der Legenden, Chroniken und der religiösen Literatur im 14. Jahrhundert begann, war Dänisch noch mit lokalen Mundarten gleichzusetzen. Eine Standardsprache gab es zur Zeit der Reformation noch nicht; sie entstand in den folgenden Jahrzehnten.

Die dänische Schriftsprache fußte im wesentlichen Grade auf die seeländischen Mundarten und die gesprochene Sprache der Bürger in Kopenhagen. Auf Seeland waren die politisch-administrativen, ökonomischen und kirchlich-religiösen Zentren Dänemarks: Kopenhagen und Roskilde. Parallel entwickelte sich der vom Latein und Deutsch stark beeinflusste seeländische Kanzleistil.

Zwei Begebenheiten waren dann entscheidend für die Geschichte Dänemarks und für die Entwicklung der dänischen Sprache: die Einführung des Buchdrucks (1482) und der lutherischen Reformation (1536). Durch die Reformation verdrängte Dänisch Latein als Kirchensprache; Latein konnte ihre herrschende Position nur in der Wissenschaft bewahren. Die Reformation sollte die Nationalsprache verstärken, und in diesem Zusammenhang war die wichtigste die Bibel von Christian III. Sie wurde 1550 veröffentlicht, und die Übersetzungsarbeit wurde nach Christian Pedersens Entwurf ausgeführt. Pedersen erwies sich als geschickter Übersetzer, hatte schon reiche Erfahrung mit der Ausarbeitung eines lateinisch-dänischen Wörterbuchs (1510) und der Übersetzung von Saxos Gesta Danorum 'Geschichte der Dänen’ (1520).

Eine misslungene Übersetzung des Alten Testaments, veröffentlicht in Leipzig 1524 als „Das Alte Testament des Königs Christian II“, spornte Pedersen an, diese Arbeit einzugehen. Christian Pedersen übersetzte dann das Neue Testament 1529, und erschuf die Grundlage für die Bibel von 1550. Wenn bei der schwedischen Übersetzung einige Zweifel zu hegen sind, ist hier klar, dass sich Pedersen auf Luthers deutsche Bibelübersetzung stützte. Christian Pedersens dänische Ausgabe hat größeren Einfluss auf die Entwicklung der dänischen Schriftsprache gehabt als irgendein anderes Buch. Pedersens Entwurf für die Übersetzung der ganzen Bibel wurde von einem königlichen Ausschuss verwendet, der vom König Christian III. mit der Ausarbeitung einer offiziellen dänischen Bibel beauftragt wurde. Der König selbst beschwor die Übersetzer, Luthers Version so genau wie möglich zu folgen. Nach vielen Revisionen und Umarbeitungen erschien die gesamte Bibel im Jahre 1550. Nach dem Auftraggeber wurde sie die Bibel von Christian III. genannt.

Die Bibel von Christian III. wurde bald sehr einflussreich, einerseits wegen ihrer konsequenten Rechtschreibung, andererseits wegen ihrer ungewöhnlich „reinen“, vom „einfachen, platten“ Dänischen inspirierten Sprache, die von den Latinismen und Germanismen gereinigt wurde (Vikør 1993: 44).

Christian Pedersen wird mit Recht als der Gründer der dänischen Schriftsprache betrachtet. In all seinen Schriften ab 1529 zeigt sich der feste Wille, „die barocke Vielfältigkeit des mittelalterlichen Schreibens mit einem geordneten System zu ersetzen“ (Haugen 1976: 324). Seine Norm war keinesfalls die Wieder- 
gabe einer bestimmten Mundart, sondern eine Fortsetzung und eine Regelung der schriftlichen Tradition in Kopenhagen. In seiner absichtlich etymologischen Schriftnorm erhielten die Wörter eine konsequente, traditionelle Form. Die wichtigsten Eigenschaften der dänischen Standardsprache wurden so mit der Bibelübersetzung 1550 festgesetzt.

Die neue Schriftnorm wurde zur Grundlage einer überlokalen gesprochenen Norm, die sich zur gesprochenen dänischen Standardsprache entwickelte. Sogar in den höchsten Kreisen waren die gesprochenen Normen zur Zeit der Einführung der Reformation nur lokal, und sie beinhalteten viele Formen, die später als vulgär gebrandmarkt wurden.

IV. Unterschiedlich von Dänemark und Schweden, hatte Norwegen eine andere historisch-politische Entwicklung. Schon im 12. Jahrhundert war das meist entwickelte Land im Norden, und verfügte über die blühende Schrifttradition. Nach dem sogenannten gammelnorsk storhetstid 'das altnorwegische goldene Zeitalter’, kam eine Niedergangsperiode für Norwegen. Das Land wurde vom „Schwarzen Tod“ verheert, und nach aufeinanderfolgenden Unionen mit Schweden (1319) und Dänemark (1380), wurde Norwegen 1450 zur dänischen Provinz. Die altnorwegische Schriftsprache verschwand allmählich, und Dänisch wurde zur Amtssprache erhoben, gebraucht in der Verwaltung und, nach der Einführung der Reformation (1536), auch in der Kirche. Die lutherische Reformation kam nach Norwegen aus Dänemark, und Latein musste in der Kirche dem Dänischen weichen (1536). Der letzte norwegische katholische Erzbischof war geflohen.

Die Reformationsliteratur kam zu den Norwegern in dänischer Sprache. Während die erste Buchdruckerei in Dänemark 1482 eingerichtet wurde, erhielt Norwegen keine bis 1643. 150 Jahre lang bekamen die Norweger (oder mussten kaufen) gedruckte Bücher aus Dänemark, und natürlich auf Dänisch. Die Bibel, der Katechismus, Psalmen- und Predigtbücher fanden Verbreitung in dänischer Sprachform.

Als die Bibel von Christian III. in Norwegen eingeführt wurde, gab es keine norwegische Übersetzung, und es sieht so aus, dass kein Norweger überhaupt an diese Möglichkeit gedacht hat. Es ist auch keine Übersetzung der Bibel ins Norwegische bis ins 19. Jahrhundert gemacht worden. So war es auch mit dem Katechismus und den Psalmenbüchern. Dänisch, das schon die Verwaltungssprache war, wurde nun auch zur Kirchen- und Schulsprache. Während in anderen Ländern die Reformation zu einer Umwälzung führte und die Bibelübersetzungen der Reformationszeit zur Grundlage der Nationalsprachen wurden, gelang es in Norwegen mit der Reformation eigentlich einer Fremdsprache, ihre Stellung zu festigen. Dänisch wurde bald zur Prestigesprache.

Am Anfang war sein sprachlicher Einfluss bescheiden, da die Bibel ein großes und teures Buch war, und zunächst nicht zur Volkslektüre wurde (Ramsfjell- 
Lundeby 1984: 27). Das einfache Volk kam in Kontakt mit der Standardsprache meistens durch die Kirche. In Norwegen war Dänisch die Kirchensprache: die Bibel, später der Katechismus und eine ganze Reformationsliteratur kamen in dieser Sprache ins Land. So erhielt „Dänisch festen Grund in der Kirche, und festigte sein Prestige unter dem einfachen Volk als das »rechte« und »göttliche« Sprache“ (Almenningen-Roksvold 1981: 50).

Norwegisch wurde im Laufe von zwei oder drei Generationen vom Dänisch als Schriftsprache ersetzt, aber überlebte in den Mundarten. Auf dem Lande sprach das Volk weiterhin Dialekt, aber in den Städten versuchten einige Norweger, Dänisch zu sprechen. Allmählich entwickelte sich unter den Norwegern eine sogenannte „Lesesprache“. Nur wenige kannten die dänischen Ausspracheregeln, und wenn sie in der Kirche vorlesen mussten, taten sie das buchstäblich, aber mit norwegischem Tonfall. Manchmal behielt man die norwegischen Lautformen und Beugungsendungen, so dass das Ergebnis eine anständige Mischung der beiden Sprachen war, „klokkerdansk“ 'Glöcknerdänisch', wie man es nannte.

Als sie 1814 ihre Unabhängigkeit von Dänemark errungen haben, konnten die Norweger in ihren Bestrebungen zwischen zwei Lösungen wählen, um eine Schriftsprache zu schaffen: entweder die Norwegisierung der dänischen Schriftsprache oder der Aufbau einer ganz neuen aufgrund der Mundarten. Die erste Lösung ergab das Dänisch-Norwegische Riksmål / Bokmål. Die zweite, die schwierigere, wurde vom genialen Linguist Ivar Aasen gewählt. Während er die neue Schriftsprache Mitte des 19. Jahrhunderts ausgearbeitet hat, testete Aasen mit Hilfe von kleineren Texten die Reaktion des Volkes. Große Erwartungen hatte er nicht; er war sich dessen bewusst, dass es noch lange dauern sollte, bis man eine künstlich aufgebaute Sprache tatsächlich verwenden könnte. Er empfahl, dass die Landsmål zuerst zur Niederschreibung von Märchen, Legenden, dann für Naturschilderungen, Novellen und Lyrik gebraucht werden sollte, und eventuell am Ende für religiöse Texte.

Im Jahre 1885 hat Stortinget, das norwegische Parlament, das Landsmål mit dem Dänisch-Norwegischen gleichgestellt. Seit 1929 heißen sie offiziell Bokmål 'Buchsprache' und Nynorsk 'Neunorwegisch'.

1904 erschien das Neue Testament auf Riksmål (Bokmål), und damit war die Übersetzung der gesamten Bibel aus den Grundtexten vollzogen (mit einer Revision 1930; zuvor erschien 1891 das Alte Testament, übersetzt aus dem Hebräischen). 1921 wurde die erste vollständige Bibelübersetzung auf Landsmål (Nynorsk) veröffentlicht (mit einer Revision 1938; zuvor das Neue Testament auf Landsmål erschien 1889, dann 1899). 1978 erschien eine neue Bibelübersetzung aus den Grundsprachen, diesmal in beiden Sprachvarianten gleichzeitig. (Übersetzung nach Luthers Prinzip, mit einer Revision 1985, diese Version hat die größte Verbreitung.) Bibel 2011 ist die neueste Ausgabe. 
V. Früher als irgendwelche andere skandinavische Sprache, war Isländisch eine herausgebildete und künstlerische Schriftsprache, und den Isländern gelang es durch Jahrhunderte hindurch ihre Sprache in der ursprünglichen Form zu bewahren. Seit dem Mittelalter haben in der Schriftsprache nur kleine Änderungen stattgefunden.

Island kam zusammen mit Norwegen unter Dänemark im Jahre 1380, aber die Isländer hatten nicht dasselbe Schicksal wie die Norweger. Isländisch konnte von den dänischen Behörden nicht unterdrückt werden, obwohl Dänisch als Amtssprache eine privilegierte Stelle hatte.

Auf Island war die literarische Tradition stark, und die Isländer gebrauchten ihre Sprache in Schrift Jahrhunderte lang ohne Änderung.

Der Buchdruck wurde 1530 in Island eingeführt, übrigens mehr als hundert Jahre zuvor Norwegen bekam eine. Der letzte katholische Bischof von Skálholt, Jón Arason, verschaffte eine Buchdruckerei. Im selben Jahr wurde er von den Dänen enthauptet. Aber mit der Druckerei waren nun die Isländer imstande, Bücher in ihrer Muttersprache zu drucken, und es wurde irgendwie selbstverständlich die Bibel zu übersetzen. Nach 10 Jahren erschien Oddur Gottskálkssons Übersetzung des Neuen Testaments (1540), das erste isländische Buch, das je gedruckt wurde. Der Übersetzer Gottskálksson entstammte einer norwegischen Familie und hatte selbst in Bergen studiert.

Das Neue Testament (1540) vereinte die mittelalterliche religiöse Terminologie mit dem gelehrten Stil des Altisländischen, und kennzeichnet sich durch den übertriebenen Gebrauch des Präsens Partizip, der Fragewörter als Relativpronomen, des Passivs auf -st usw. Das Hauptmerkmal ist aber die Bestrebung, die deutschen und dänischen Originale getreu, d. h. Wort für Wort zu übersetzen, und gleichzeitig dem Bedarf der Leser gerecht zu werden. Viele Wörter sind auch heutzutage im Gebrauch. Die Rechtschreibung war von der dänischen beeinflusst, war sogar, außer dem Zeichen $b$, auf die dänischen Buchstaben eingeschränkt (also keine Akzentzeichen, dänisches ø für das isländische $\ddot{o}$, und $d$ für $ð$ ), außerdem starker dänischer Einfluss auch in der Grammatik, z. B. -e für $-i$ in Endstellung.

Die Übersetzung des Alten Testaments ins Isländische war nur der Anfang einer neuen Entwicklung, die dann eine ganze Reihe von Büchern und Übersetzungen im Land zur Unterstützung der Reformation hervorbrachte. Die Isländer aber ließen sich nicht so leicht von den Reformatoren überzeugen, ihr Widerstand wurde erst rund 1550 endgültig gebrochen.

Einige Jahrzehnte nach der Lutherschen Reformation erschien eine vollständige isländische Übersetzung der Bibel (1584), die nach dem Übersetzer, Bischof Guðbrandur Porláksson, „Guðbrandsbiblia“ genannt worden ist. Diese Bibel wurde auf derselben Druckerei, die von Jón Arason geschafft wurde, gedruckt, und ebenda kamen noch eine Menge andere Bücher mit religiösem 
Inhalt in den darauffolgenden Jahren zustande. (Die meisten Lehnwörter in den religiösen Schriften blieben aber nur Literaturwörter, sie fanden nie Eingang in die gesprochene Sprache.)

Damit wurde der isländischen Schriftsprache eine zusammenhängende Tradition und Kontinuität gesichert, und Isländisch war ins neue Zeitalter hinübergerettet. Isländisch blieb die Sprache der Kirche, während sich Dänisch als Amtssprache in der Verwaltung durchgesetzt hat.

1944 wurde Island unabhängig und Isländisch zur einzigen offiziellen Sprache im Lande.

VI. Wie Isländisch, hatte Färöisch ihren Ursprung in einer südwestnorwegischen Mundart, aber im Gegensatz zu Isländisch, verfügte sie über eine kurze und bescheidene Schrifttradition. Die Färöer-Inseln wurden nie zu einem mittelalterlichen Literaturzentrum wie die benachbarte Inselgemeinschaft, und es gab auch wenige Dokumente des altnordischen Zeitalters. Als die Dänen die Verwaltung der Färöer-Inseln übernahmen, haben sie ihre eigene Sprache verwendet, sowohl schriftlich als auch mündlich, wie in Norwegen. Färöisch überlebte nur als gesprochene Sprache in den Mundarten, wie Norwegisch.

Bei der Reformation hatten die Färinger, d. h. die Bürger der Färöer-Inseln, dasselbe Schicksal wie die Norweger. Die Inseln waren längst unter Dänemark, und Dänisch trat als Bibel- und Kirchensprache an die Stelle des Lateins, und nicht die Muttersprache. Durch die Verwaltung drang die dänische Schriftsprache auch in die anderen Bereiche der Gesellschaft ein und schlug dort tiefe Wurzeln. Auch politisch war Färöisch zu schwach dazu. Dann gab es keine färöische Schriftnorm im Mittelalter, im Gegensatz zur reichen Tradition des Isländischen.

Morpho-syntaktisch stabilisierte sich Färöisch an einer Zwischenstufe zwischen Isländisch und Kontinentalskandinavisch, phonetisch aber unterscheidet sich auffallend von den anderen skandinavischen Sprachen, vor allem in der Entwicklung einer Reihen von Zwielauten aus den alten Vokalen. Die färöische Rechtschreibung wurde 1846 vom Geistlichen und Volkskundler Venceslaus Ulricus Hammershaimb ausgearbeitet. Für ihn war der geschichtliche Zusammenhang am wichtigsten, folglich nahm er die altnordische Schriftsprache als Muster. Daher auch die Ähnlichkeit mit der isländischen Schriftsprache. In der Staatskirche, die lange Zeit vom Dänisch dominiert wurde, konnte sich Färöisch erst später, nämlich im 20. Jahrhundert durchsetzen.

Vikør sieht den Grund darin, dass Färöisch traditionell nicht als eine separate Sprache betrachtet wurde, und man ging davon aus, dass die Färinger Dänisch verstanden. Merkwürdigerweise kam ein Teil des Widerstands von der lokalen Bevölkerung selbst. Sobald sie sich an Dänisch als Religionssprache angewöhnt haben, „,betrachteten sie ihre eigene Sprache als zu platt und würdelos um in jenen hochliegenden Sphären akzeptiert zu werden“ (Vikør 1993: 107). 
Das Jahr 1948 brachte das Lov om Færøernes Hjemmestyre 'Gesetz über Selbstverwaltung', das den Färöern Autonomie und Selbstbestimmungsrecht gab und Färöisch mit Dänisch gleichstellte. 1949 erschien die Bibel zum ersten Mal in färöischer Sprache in der Übersetzung des Missionars Victor Danielsen. 1974 wurde eine kleine Revision, leichte Überarbeitung unternommen. Zuvor wurde das Neue Testament in färöischer Sprache schon 1937 veröffentlicht.

VII. Der Überblick über die ersten vollständigen Übersetzungen der Bibel in die skandinavischen Sprachen zeigt uns ein sehr unterschiedliches Bild.

Die Reformation war ein Wendepunkt in der Geschichte, führte zu einer Umwälzung in vielen Ländern, indem sie die sozio-kulturelle Entwicklung dieser begünstigte, aber in einigen Fällen im Gegenteil, bremste. In Deutschland, Schweden und Dänemark wurde Latein aus der Kirche verdrängt und ersetzt mit der Landessprache. In diesen Ländern wurden die ersten Bibelübersetzungen die Grundlage für die nationalen Schriftsprachen und der Ansporn, die Beschleunigung für die nationalen Kulturen. Das technische Mittel zur Verbreitung lieferte die Erfindung des Buchdrucks.

Dagegen hatte die Reformation in Norwegen und auf den Färöer-Inseln verhängnisvolle Folgen. Eine fremde Schriftsprache, die dänische, schlug tiefe Wurzeln. Norwegen war zu geschwächt und zu zerspaltet um Widerstand zu leisten. Die ersten Bibelübersetzungen in der Landessprache (Norwegisch, in den beiden Schriftformen Bokmål und Nynorsk, beziehungsweise Färöisch) sind dann erst in der modernen Zeit erschienen.

Die Lage des Isländischen nimmt in diesem Zusammenhang eine Zwischenstellung ein. Hier war die Schriftsprache schon längst entwickelt, verfügte über eine reiche Tradition und Kontinuität, um von einer fremden Schriftsprache unterdrückt zu werden. Die Bibel in der Landessprache erschien folglich schon im Spätmittelalter.

\section{Literaturverzeichnis}

Almenningen, Olaf-Roksvold, Thore-Sandøy, Helge-Vikør, Lars. S. 1981. Språk og samfunn gjennom tusen år. Ei norsk språkhistorie. Oslo-Bergen-Tromsø: Universitetsforlaget.

Haugen, Einar. 1978. Språket: en sosiolingvistisk profil. In: Papazian, Erik-Ystad, Vigdis (red.). 1978. Språk og tekst. Synspunkter på språk, litteratur og samfunn. Oslo-Bergen-Tromsø: Universitetsforlaget.

Haugen, Einar. 1976. The Scandinavian Languages. An Introduction to their History. London-Cambridge: Faber and Faber Limited. 
Jahr, Ernst Håkon.1992. Innhogg i nyare norsk språkhistorie. Oslo: Novus Forlag. Jahr, Ernst Håkon. 1994. Utsyn over norsk språkhistorie etter 1814. Oslo: Novus Forlag.

Leitre, Arild-Lundeby, Einar-Torvik, Ingvald. 1990. Språket vårt før og nå. Bokmål, 3. utgave, 4. opplag, Oslo: Gyldendal Norsk Forlag.

Martinsen, Osvald-Lind, Asbjørn-Raastad, Frederik. 1983. Fra rune til slang. Morsmålskunnskap. Bokmål. Språkhistorie. Norrønt. Islandsk. Dialekter. Sosiolekter m.m. Oslo: J. W. Cappelens Forlag a.s.

Papazian, Erik-Ystad, Vigdis (red.). 1978. Språk og tekst. Synspunkter på språk, litteratur og samfunn. Oslo-Bergen-Tromsø: Universitetsforlaget.

Ramsfjell, Berit Sagen-Vinje, Finn, Erik. 1984. Språkkunnskap. Språkhistorie. Norrønt. Nyislandsk. Dialekter. 2. utgave, Bokmål, Oslo: Aschehoug.

Sandøy, Helge. 1977. Island: Historia ligg gjømt i språket. In: Vikør, Lars S.: Språkpolitikk på fem kontinent. Eit oversyn og ei jamføring. Oslo: Det Norske Samlaget, 75-98.

Sjögren, Gunnar. 1949. Om språket i de svenska bibelöversättningarna 15261541. Lund: C. W. K. Gleerup.

Torp, Arne-Vikør, Lars S. 1993. Hovuddrag i norsk språkhistorie. Oslo: Ad Notam Gyldendal.

Vikør, Lars S. 1977. Språkpolitikk på fem kontinent. Eit oversyn og ei jamføring. Oslo: Det Norske Samlaget.

Vikør, Lars S. 1993. The Nordic Languages. Their Status and Interrelations. Oslo: Novus Press (Nordic Language Secretariat, Publication no. 14) 